\title{
Assessment of Functioning among Nigerians with Schizophrenia in a Tertiary Health Facility: Influence of Psychopathology, Socio-demographic and Treatment Factors
}

\author{
Elizabeth Adebola Campbell $^{1}$ Andrew Toyin Olagunju ${ }^{1,2}$ - Olatunji Francis Aina ${ }^{1,2}$. \\ Raphael Emeka Ogbolu ${ }^{1}$
}

Received: 27 June 2014/ Accepted: 5 March 2015/Published online: 3 April 2015

(C) Springer India Pvt. Ltd. 2015

\begin{abstract}
To investigate the profile of functional impairment and the influence of psychopathology with other determinants among individuals with schizophrenia in a Nigerian tertiary health facility. The study participants consisted of one hundred adults with schizophrenia. Eligible participants were recruited using systematic random sampling technique. All participants were interviewed with questionnaire to elicit socio-demographic and treatment related information. This was followed by interview with brief psychiatric rating scale (BPRS) and the global assessment of functioning (GAF) to assess for psychopathology and impairment in functioning respectively. Analyses of data were done using SPSS-16. The mean age of the participants was $33.87( \pm 11.6)$ years and a little above half $(52 \%)$ of the participants were female. Sixty (60\%) subjects had active psychopathology using BPRS cut-off score of $>10$; while participants with duration of illness of more than 10 years $\left(X^{2}=5.59, p=0.018\right)$ and previous relapse $\left(X^{2}=6.34, p=0.012\right)$ were more likely to have psychopathology. Overall, a little over two-thirds (69\%) had impairment following administration of GAF. Mild impairment was observed in $23 \%$ of the participants, moderate impairment in 19 and up to $18 \%$ of participants had severe impairment due to psychopathology. Subjects with lesser education, duration of illness of more than 10 years, extrapyramidal side effects (EPSE), previous relapse and psychopathology had significant levels of
\end{abstract}

Andrew Toyin Olagunju

aolagunju@unilag.edu.ng; andyolagus@yahoo.com

1 Department of Psychiatry, Lagos University Teaching Hospital, PMB 12003, Lagos, Nigeria

2 Department of Psychiatry, College of Medicine, University of Lagos, PMB 12003, Lagos, Nigeria impairment $(\mathrm{p}<0.05)$. However, it was education (Odds ratio $[\mathrm{OR}]=0.060 ; 95 \%$ Confidence interval $[95 \% \mathrm{CI}]=$ $0.055,0.770 ; \mathrm{p}=0.031$ ), psychopathology (OR 5.265; $95 \%$ CI 2.744, 13.626; $\mathrm{p}<0.001$ ) and EPSE (OR 0.183; $95 \%$ CI $0.049,0.939 ; \mathrm{p}=0.043$ ) that were independently associated with impairment in functioning following logistic regression to control for effects of confounders. In this study, varied degrees of impairment in functioning were prevalent among individuals with schizophrenia under treatment. However, those with lesser education, EPSE, active psychopathology and longer duration of illness were more likely to be impaired. Thus, better attention to the management of active psychopathology and prevention of extra-pyramidal side effects are indicated in order to promote improve functioning in schizophrenia. In addition, promotion of recovery oriented psychosocial services with need-based rehabilitation program for individuals with schizophrenia is advocated. There is need for more research on predictors of functioning towards promotion of evidence based rehabilitation.

Keywords Functioning - Impairment · Psychopathology · Schizophrenia

\section{Introduction}

Impairment in functioning (that includes bodily function problems, restriction of activities, disabilities and problems in the environment, environmental barriers) is a common accompaniment of schizophrenia which has been linked with considerable distress in those affected and in their relatives [1-3]. This is because such impairment often results in difficulties in activities of daily living as well as poor interpersonal relationships; and in turn, contributes to 
low levels of productivity with high rates of unemployment among others [4-6]. Despite the striking nature of the psychotic symptoms of schizophrenia, impairments with its associated problems are more relevant in the expression of its economic costs [7]. For instance, the study on global burden of diseases reported that schizophrenia accounts for significant fraction of disability-adjusted life years as well as years lived with disability [8].

In spite of the above-mentioned issues on schizophrenia, relatively less attention was paid to the management of the disabling impacts of schizophrenia until recently. This was exemplified in the construct of the traditional mental health treatment services as they were mainly focused on reduction of clinical symptoms (positive, negative, and disorganized), their direct consequences and on relapse prevention [9]. Unfortunately, such approach has been observed to be limited at addressing the significant impairment experienced by people with schizophrenia. For example, while antipsychotic drugs were observed to be effective at alleviating some of the distressing symptoms of schizophrenia and reduce relapse rates; their roles in providing sufferers with coping mechanisms and practical skills to aid rehabilitation were limited [10]. Despite mixed findings with respect to the outcome in schizophrenia; attainment of recovery is receiving better attention, as it is widely accepted that recovery is possible to the extent of absence of all symptoms and disabilities [9].

While the estimation of the burden of functional impairment in schizophrenia may be useful in planning intervention services; information on key determinants of functioning in those affected as done in this work has positive implications for recovery as well as evolvement of preventive measures and the alleviation of caregivers' burden. The latter is important in developing contexts, where up to $90 \%$ of individuals with schizophrenia have been observed to live with their families [11], who play key roles in their care and complement the inadequacies of the existing mental health facilities $[12,13]$.

A review of literature suggests that the impairments described in relation to schizophrenia are largely homogeneous across different contexts [14, 15]. However, a number of environmental, cultural, psychosocial and treatment related factors among others may determine the differences in outcome and the expression of functional impairments in schizophrenia across contexts. Such factors include frequency of relapse, disability compensation, opportunities, residential support, poor treatment compliance, elements of attitudes and stigma among others [16]. Regrettably, despite the aforementioned, rehabilitation programmes are currently underfunded, less driven by research based evidence and largely unavailable in many developing countries $[6,17]$.
To this end, the care of people with schizophrenia poses a major challenge in many contexts, however much more in the developing countries as indicated above. This is further compounded by issues like out-of the pocket payment system, poor health insurance coverage and belief system among others [18, 19]; thereby, contributing to the gap noticeable in mental health treatment. This study is set to investigate the profile of functional impairment and the influence of psychopathology with other determinants among individuals with schizophrenia in a Nigerian tertiary health facility. It is postulated that individuals with schizophrenia would experience varied degrees of impairment in functioning and the presence of psychopathology, socio-demographic and treatment related factors would constitute important determinants.

\section{Methods}

\section{Study Location and Participants}

This is a descriptive cross-sectional study resulting from secondary analyses of data collected in a project conducted in the psychiatric outpatient clinic of Lagos University Teaching Hospital (LUTH) Lagos. LUTH is a federal government owned tertiary health institution with referrals from peripheral health facilities in Lagos state and its environs. The study participants consisted of one hundred adults with schizophrenia attending outpatient clinics. Diagnosis of schizophrenia was validated by two psychiatrists based on International Classification of Disease tenth edition following clinical history and assessment of symptoms about the patients [20]. The sample size adopted was based on estimation in line with sample size calculation formula [21] and study was carried out over a period of 6 months (July-December 2013). Patients' recruitment was done from eligible clinic attendees, made up of an average of 20-50 individuals with mental illnesses per clinic.

Eligible participants were consenting individuals with schizophrenia, who were currently on medication and had been on treatment for at least 6 months. Those with chronic co-morbid diagnoses like diabetes, hypertension, and stroke among others were excluded. Subjects' selection was by systematic random sampling technique, having generated serial number and sampling interval from patients attendance register. Participant's recruitment was done on each clinic day using the record list of patients in attendance. The first participant was chosen from the patients' list by balloting from within the list of those present and the subsequent participants were an addition of the sampling interval to the first number. For the participants that did not meet the inclusion criteria or declined consent; 
the next participant in the list was recruited. Overall, five patients declined consent.

\section{Ethical Issues and Study Procedure}

The ethical approval was obtained from the Health Research and Ethics committee of the hospital before commencement of the study, while written informed consent was obtained from each subject with their relatives and confidentiality was maintained. The interviewers explained the nature and objectives of study to patients and their relatives. They were also assured that consent can be declined at any point of the study without any negative consequence on the patient's treatment. Consenting participants were interviewed using socio-demographic and clinical data questionnaire to gather data such as age, sex, religion, highest level of education, occupation, and employment status. Clinical information about the subjects' illness was also got from the patients' case notes and these included duration of illness, age of onset, previous admissions and number of relapses, type of medication and presence of extrapyramidal side effects (EPSE). The clinical variables of interest in this work were in line with the information that are always requested from all patients (required information in clerking guideline) and included in their case notes. However, ascertainment and clarification of information from patients and their relatives were done where necessary. In addition, independent assessment of EPSE was done by the researchers (psychiatrists) using the guide from abnormal involuntary movement scale where indicated. Prior training was also done to improve the inter-rater validity. This was followed by brief psychiatric rating scale (BPRS; [22]) to assess presence and severity of psychopathology.

A BPRS cut-off score of $>10$ was used in line with previous findings in this environment [23] validating this cut-off score for the presence of prominent psychotic symptoms. Subsequently, global assessment of functioning (GAF) [24] was used to assess the overall functioning among the participants and the resulting scores were categorised during analyses. Participants with scores between 81 and 100 were grouped as having no impairment, while those with scores ranging from of $0-80$ indicating mild impairment to severe impairment due to psychopathology were grouped as impaired [23, 24]. For better analyses of the determinants of impairment among the participants, scores on the GAF were dichotomised, with those participants having no impairment and those who were mildly impaired grouped as no impairment, while those with moderate and severe impairments were categorised as having impairment.

Data were analysed using the 16th version of the Statistical Package for Social Sciences [25] to generate frequency tables, percentages and other relevant statistics. Chi square test was used to establish associations between variables and logistic regression analyses were used to ascertain independent associations between relevant variables. CI of $95 \%$ which allows for $5 \%$ sampling error, at significance level less than or equals to 0.05 was used.

\section{Results}

\section{Socio-demographic/Clinical Characteristics of Participants}

In this study, majority of the subjects were females (52\%) and half of them $(50.0 \%)$ were 30 years and below. The mean age of participants was $33.87 \pm 11.6$ years. Majority, $(71.0 \%)$ were single; while with respect to education, $14 \%$ had primary school education, $38 \%$ had secondary school education and the remaining $48 \%$ had one form of tertiary education or the other. In the course of analyses, educational status was further classified as follows primary school (lower education) and post-primary school (higher education). Majority ( $82 \%$ ) were Christians while others were Muslims. Overall, $23 \%$ were schooling while $4 \%$ of the participants were retired. $22 \%$ of the participants were employed and $27 \%$ of those employed were engaged in highly skilled jobs.

In terms of clinical characteristics, about a fifth of the subjects $(19.0 \%)$ were diagnosed with schizophrenia before their 20th birthday, a little less than half of them $(48.0 \%)$ have had the diagnosis for more than 5 years and $22.0 \%$ for more than 10 years. A third $(33.0 \%)$ was diagnosed in the first episode, while $41.0 \%$ had less than 3 relapses. Half of the participants $(50.0 \%)$ had never been admitted for their mental health issues, while 39 (39.0\%) had up to 2 previous admissions. Typical and atypical antipsychotics were evenly used (50.0\% respectively) with EPSE present in about a quarter $(24.0 \%)$. With regards to psychopathology, Fig. 1 indicates that sixty $(60 \%)$ of the subjects had psychopathology using BPRS. A number of factors were associated with psychopathology among subjects. In this regard, subjects with duration of illness of more than 10 years had significant level of psychopathology $\left(\mathrm{X}^{2}=5.594, \mathrm{p}=0.018\right)$ and history of previous relapse was statistically associated with psychopathology $\left(X^{2}=6.340, p=0.012\right)$ as $68.7 \%$ of those with previous relapse had psychopathology compared with $42.4 \%$ of those without.

\section{Pattern of Impairment in Participants}

Table 1 shows that a little over two-thirds (69\%) of them had impairment following administration of GAF. Mild 


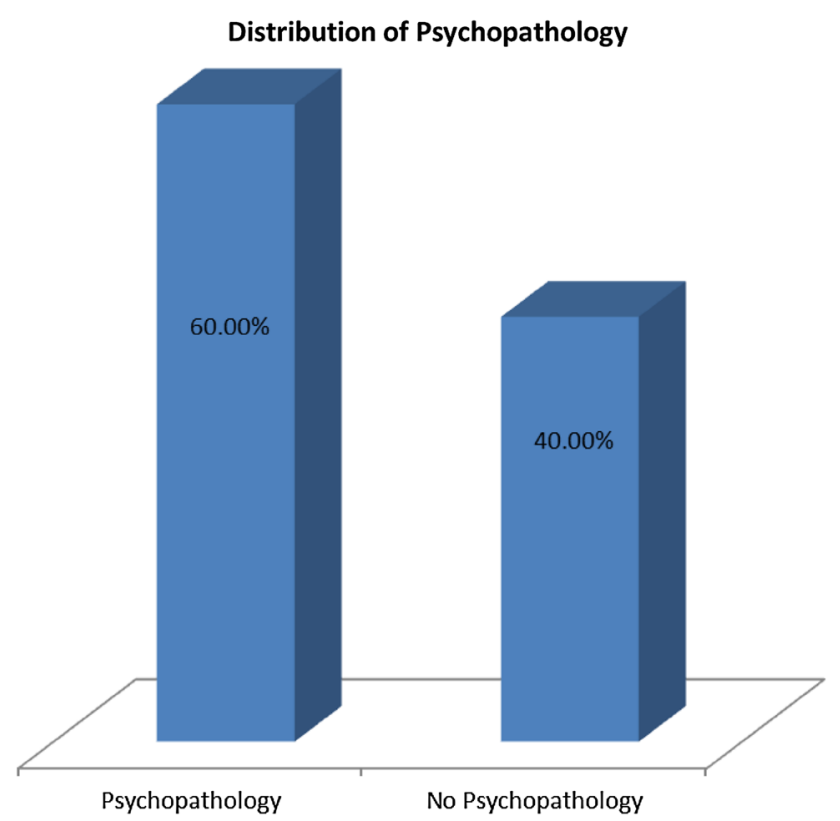

Fig. 1 Distribution of psychopathology based on BPRS score

Table 1 Distribution of impairment among subjects

\begin{tabular}{lcr}
\hline & Frequency $(\mathrm{n})$ & $(\%)$ \\
\hline Impairment & & \\
No impairment (81-100) & 31 & 31 \\
Mild impairment (61-80) & 23 & 23 \\
Moderate impairment (51-60) & 19 & 19 \\
Serious impairment (31-50) & 9 & 9 \\
Impairment due to psychopathology (1-30) & 18 & 18 \\
Total & 100 & 100 \\
\hline
\end{tabular}

impairment was observed in $23 \%$ of the participants and up to $18 \%$ of participants had impairment due to psychopathology.

\section{Determinants of Impairment in Participants}

Table 2 shows the relationship between psychopathology and impairment in the subjects. Most of the subjects with current psychopathology (93.3\%) had impairment as compared to $32.2 \%$ of those without psychopathology who had impairment. Psychopathology $\left(X^{2}=43.91\right.$, $\mathrm{p}<0.001)$ was statistically associated with impairment. In addition, significant relationship between a few socio-demographic with clinical characteristics and impairment in the subjects was noted. For instance, subjects with only primary school education $\left(X^{2}=5.51, p=0.019\right)$ and those with a duration of illness of more than 10 years $\left(X^{2}=8.11, p=0.004\right)$ had significant level of impairment. Impairment was also statistically associated with
EPSE $\left(X^{2}=5.23, p=0.021\right)$, and previous relapse $\left(X^{2}=6.95, p=0.007\right)$.

\section{Logistic Regression of Factors Associated with Psychopathology and Impairment}

Following logistic regression to control for effects of confounders, both previous relapse [Odds ratio $(\mathrm{OR})=$ 2.24; $95 \%$ confidence interval $(\mathrm{CI})=0.894,5.589 ; \mathrm{p}=$ 0.085 ] and duration of illness (OR 2.732; $95 \%$ CI 0.791 , 9.439; $\mathrm{p}=0.112$ ) were not independently associated with psychopathology.

On the other hand, education (OR 0.060; $95 \%$ CI [95\% CI] $0.055,0.770 ; \mathrm{p}=0.031$ ), psychopathology (OR 5.265; $95 \%$ CI 2.744, 13.626; $\mathrm{p}<0.001$ ) and EPSE (OR 0.183; $95 \%$ CI $0.049,0.939 ; \mathrm{p}=0.043$ ) were independently associated with impairment. See Table 3.

\section{Discussion}

The care of mental illnesses and in particular schizophrenia is evolving around 'recovery-based' treatment approach. Such approach is arguably preferred because of the need to address the disabling impacts of these mental disorders on those affected. In this study, some findings that are of relevance to the development of such 'recovery-based' clinical and rehabilitative interventions were observed. For instance, this study observed significant burden of impairment in functioning, albeit of varied degrees from one individual with schizophrenia to the other. Additionally, those with lesser (primary school) level of education, longer duration of illness, previous relapse, presence of EPSE and active psychopathology were more likely to be impaired in functioning. However, presence of active psychopathology, lesser (primary) education, and more than 10 years duration of illness were the only independent correlates of impairment in functioning following control for effects of confounders.

\section{Socio-demographic Profile}

Aside from the above, some issues of interest border on the pattern of socio-demographic profile of the participants when compared to other similar studies. In this study, majority of the subjects were young and the illness also began at a relatively young age. This is consistent with the findings in previous studies $[26,27]$ and confirms the fact that schizophrenia usually begins at an early age [28]. Similar age range has been reported in this environment $[29,30]$, however, reported data on age are usually interpreted cautiously considering the widespread of illiteracy among majority of patients and their caregivers in this 
Table 2 Relationship between clinical/demographic characteristics and psychopathology with impairment
Table 3 Logistic regression analysis of correlates of psychopathology and impairment among participants

\begin{tabular}{|c|c|c|c|c|c|}
\hline Variables & Impairment & No impairment & $X^{2}$ & $\mathrm{df}$ & $\mathrm{p}$ value \\
\hline \multicolumn{6}{|l|}{ Education ${ }^{\circledR}$} \\
\hline Primary & $11(78.6)$ & $3(21.4)$ & & & \\
\hline Post primary & $35(40.7)$ & $51(59.3)$ & 5.512 & 1 & $0.019^{\uparrow}$ \\
\hline \multicolumn{6}{|c|}{ Duration of illness } \\
\hline$\leq 10$ years & $30(38.5)$ & $48(61.5)$ & & & \\
\hline$>10$ years & $16(72.7)$ & $6(23.3)$ & 8.111 & 1 & $0.004 *$ \\
\hline \multicolumn{6}{|l|}{ EPSE } \\
\hline No & $29(39.2)$ & $45(60.8)$ & & & \\
\hline Yes & $17(65.4)$ & $9(36.4)$ & 5.315 & 1 & $0.021 *$ \\
\hline \multicolumn{6}{|c|}{ Previous relapse } \\
\hline No & $9(27.3)$ & $24(72.7)$ & & & \\
\hline Yes & $37(55.2)$ & $30(44.8)$ & 6.954 & 1 & $0.007 *$ \\
\hline \multicolumn{6}{|c|}{ Psychopathology } \\
\hline No & $45(75.0)$ & $39(97.5)$ & & & \\
\hline Yes & $45(75.0)$ & $15(25.0)$ & 43.908 & 1 & $<0.001^{\uparrow}$ \\
\hline
\end{tabular}

$X^{2}$ Chi square; $d f$ degree of freedom; $p$ level of significance, $* \mathrm{p}<0.05 ;$ EPSE extrapyramidal side effect; ${ }^{\uparrow}$ Fisher exact test; ${ }^{\circledR}$ Educational status was classified as follows primary (lower education) and post-primary (higher education)

\begin{tabular}{lrlllllll}
\hline Variables & \multicolumn{1}{l}{$\beta$} & SE & Wald & df & Sig & Exp $\beta$ & \multicolumn{2}{c}{$95.0 \%$ CI } \\
\cline { 6 - 9 } & & & & & & & Lower & Upper \\
\hline Psychopathology & & & & & & & & \\
$\quad$ Duration of illness & 1.005 & 0.633 & 2.525 & 1 & 0.112 & 2.732 & 0.791 & 9.439 \\
Previous relapse & 0.804 & 0.468 & 2.960 & 1 & 0.085 & 2.235 & 0.894 & 5.589 \\
Constant & -2.115 & 0.895 & 5.578 & 1 & 0.018 & 0.121 & & \\
Impairment & & & & & & & & \\
Psychopathology & 5.265 & 1.353 & 15.139 & 1 & $\mathbf{0 . 0 0 0}$ & 1.933 & 2.744 & 13.626 \\
Education & -2.820 & 1.305 & 4.466 & 1 & $\mathbf{0 . 0 3 1}$ & 0.060 & 0.005 & 0.770 \\
Duration of illness & 1.000 & 0.822 & 1.481 & 1 & 0.224 & 2.719 & 0.543 & 13.066 \\
EPSE & 1.578 & 0.781 & 4.506 & 1 & $\mathbf{0 . 0 4 3}$ & 0.183 & 0.049 & 0.939 \\
Previous relapse & 1.265 & 0.830 & 2.320 & 1 & 0.128 & 3.542 & 0.696 & 6.160 \\
Constant & 7.728 & 2.250 & 5.656 & 1 & 0.017 & 0.001 & & \\
\hline B & & & & & &
\end{tabular}

Bold values indicate statistically significant at $p<0.05$

Wald wald Chi square, $S E$ standard error, $d f$ degree of freedom, sig. level of significance, $\operatorname{Exp} \beta$ odds ratio,

CI confidence interval

setting. In contrast to findings of some other studies [26, 27, 29], preponderance of females was noted in this study. However, Yusuf et al. [31] reported a preponderance of female patients. The similarity between this study and the later could be due to pattern of better health seeking behaviour of females in the two study areas (South west Nigeria).

It is not surprising that majority of the studied participants were single because the onset of schizophrenia is commonly in early life and could have prevented attainment of major life achievements in which marriage is one. There are also a lot of taboos in our society concerning mental illness; even when the subjects are free from symptoms, they find it difficult getting suitor because of the stigma attached to mental illness [32]. A significant level of unemployment was observed among the participants as close to eight in every ten of them were unemployed. Comparatively, this observed rate of unemployment is about four-fold the national rate of unemployment (23.9\%; [33]). This finding is not surprising because of its congruence to what has been seen among people with severe mental illness in other studies [16, 32]. It is however interesting that while the high level of unemployment in this study may be related to presence of considerable impairment among the participants; it is also tenable to consider the interplay of social exclusion due to stigma in people 
with mental illness as a possible reason for the presence of unemployment among those with no impairment.

\section{Psychopathology and Impairment with Associated Factors}

Overall, close to two-thirds of the subjects were noted to have active psychopathology despite being on treatment. This finding further buttressed the fact that schizophrenia is a chronic illness with frequent relapses and many of those affected may still have symptoms after the acute phase of the illness [34, 35]. Similarly, previous studies have fielded high figures for prevalence of psychopathology among individuals with schizophrenia despite antipsychotic use $[24,25]$. The use of different research instruments, difference in length of stay, and duration as well as type of treatment are few of the posited reasons for the variance in the discrete figures fielded for the burden of psychopathology between the cited studies and this one.

In a similar vein, two-thirds of the subjects in this study had impairment using GAF, which is consistent with existing literature. For instance, a multicentre study conducted among 524 patients with schizophrenia on oral and long acting olanzapine using a different instrument reported that majority of their subjects had impairment with a fifth of them having good level of functioning [25]. Other studies have reported similar range of figures among people with schizophrenia with regards to functioning using the same instrument [36], or with the use of different instrument [37]. Generally, recent findings suggest a relatively better level of functioning in a large proportion of individuals with schizophrenia when compared to the traditional Kraepelin classification [26], and the difference is possibly due to advancement in treatment.

In this study, a number of factors were associated with impairment that includes presence of psychopathology. Previous research works on schizophrenia have indicated strong linkage between increasing frequency of symptoms with poorer level of functioning. Arguably, there is established relationship between functioning and lower severity of schizophrenic symptoms, greater levels of productive activity and higher scores on other health-related QOL measures [37]. Similarly, positive correlation between reduced psychopathology and increasing GAF score i.e. better functioning has been reported [38]. This may not be farfetched because the GAF scale recognizes psychopathology in its construct, with presence of psychopathology usually accrues lower GAF score. The observation that those with less than a secondary school education were more likely to report lower level of functioning is consistent with earlier study [36]. The linkage of better education (post primary) with improved functioning is explainable by the positive effect of education on psychological functioning as noted in existing literature [39]. Again, better education may promote good health seeking behaviour as well as enhance improved adjustment to the illness with functioning.

However, contrary to earlier findings, previous relapse and longer duration of illness were found to correlate with lower level of functioning [36]. Aside from these mentioned clinical factors, the presence of EPSE was linked with the likelihood of impairment of function in this study. This is because EPSE might colour the expression of residual psychopathology, and the resulting involuntary movements could be distressful to patients and their relatives as well as impair patients functioning. This has significant clinical implication in settings where EPSE are common, and in particular where typical antipsychotics are still largely used due to poverty [40].

\section{Limitations}

A number of shortcomings were identified in this study. For example, its cross sectional design limits the strength of inferences that could be made on the causal relationship between impairment in functioning and identified associated factors. In addition, the clinical setting of the study suggests a limitation in terms of generalizability to all population of individuals with schizophrenia in the community. Again, the weakness of GAF in the assessment of functioning across multiple domains, especially in comparison to other instruments and lack of use of structured instruments to assess EPSE may constitute potential shortcomings of the present study that should be addressed in future study.

\section{Conclusion}

In conclusion, the findings from this study will help to direct development of evidence based psychosocial intervention targeted towards recovery in people with schizophrenia based on what is known on determinants of functioning. In this respect, this study suggests that presence of psychopathology and extra-pyramidal side effects were key independent determinants of functioning. Thus, active management of psychopathology with associated impairment as well as preventive strategy against EPSE are implied among individuals with schizophrenia for improved outcome in functioning. Given the foregoing, despite not directly investigated in this study, optimal with rational use of therapeutic agents are indicated. In addition, the promotion of recovery oriented psychosocial services with need-based rehabilitation programs for individuals with schizophrenia is advocated Furthermore, there is need for more research on predictors of functioning using 
longitudinal study design to ensure informed rehabilitative programs for schizophrenia.

Disclosure The authors wish to report no commercial or proprietary interest in the concept discussed in this article.

\section{References}

1. McGurk SR, Lee MA, Jayathilake K, Meltzer HY. Cognitive effects of olanzapine treatment in schizophrenia. Medscape Gen Med. 2004;6:27.

2. Goldacre M, Shiwak R, Yates D. Estimating incidence and prevalence of treated psychiatric disorders from routine statistics: the example of schizophrenia. J Epidemiol Comm Health. 1994; 48:318-22.

3. World Health Organization. Towards a common language for functioning, disability and health, ICF. Geneva: World Health Organization; 2002.

4. Lauriello J, Lenroot R, Bustillo JR. Maximizing the synergy between pharmacotherapy and psychosocial therapies for schizophrenia. Psychiatr Clin North Am. 2003;26:191-211.

5. Lenroot R, Bustillo JR, Lauriello J, Keith SJ. Integrated treatment of schizophrenia. Psychiatric Serv. 2003;54:1499-507.

6. Awad AG, Voruganti LN. The burden of schizophrenia on caregivers: a review. Pharmacoeconomics. 2008;26:149-62.

7. Leung WW, Bowie CR, Harvey PD. Functional implications of neuropsychological normality and symptom remission in older outpatients diagnosed with schizophrenia: a cross-sectional study. J Int Neuropsychol Soc. 2008;14:479-88.

8. Rössler W, Salize HJ, Os J, Riecher- Rössler A. Size of burden of schizophrenia and psychotic disorders. Eur Neuropsychopharmacol. 2005;15(4):399-409.

9. Harvey PD, Bellack AS. Toward a terminology for functional recovery in schizophrenia: is functional remission a viable concept? Schizophr Bull. 2009;35(2):300-6.

10. Jablensky A, Sartorius N, Ernberg G, Anker M, Korten A, Cooper JE, Day R, Bertelsen A. Schizophrenia: manifestations, incidence and course in different cultures. A world health organization ten-country study. Psychol Med Monogr Suppl. 1992;20: 1-97.

11. World Health Organization. Mental health atlas. Geneva: World Health Organization. www.globalatlast.who.int/globalatlast/default. asp. Accessed 6 Jan 2010.

12. World Health Organization. Nations for mental illness: schizophrenia and public health. Geneva: World Health Organization. http://www. who.int/media/en/55pdf. Accessed 6 Jan 2010.

13. Patel V, Cohen A, Thara R, Gureje O. Is the outcome of schizophrenia really better in developing countries? Rev Bras Psiquiart. 2006;28(2):149-52.

14. Harvey PD, Helldin L, Bowie CR, Heaton RK, Olsson A, Hjärthag F, Norlander T, Patterson TL. Performance-based measurement of functional disability in schizophrenia: a crossnational study in the United States and Sweden. Am J Psychiatry. 2009;166:821-7.

15. Green MF, Schooler NR, Kern RD. Evaluation of functionallymeaningful measures for clinical trials of cognition enhancement in schizophrenia. Am J Psychiatry. 2011;168:400-7.

16. Rosenheck R, Leslie D, Keefe R. Barriers to employment for people with schizophrenia. Am J Psychiatry. 2006;163:411-7.

17. Puri BK, Hall AD. Management of schizophrenia. In: Kazdin AE, editor. Revision notes in psychiatry. 2nd ed. New York: Schizophrenia; 2004. p. 378-9.
18. Suleiman TG, Ohaeri JU, Lawal RA, Orija OB. Financial cost of treating out-patients with schizophrenia in Nigeria. B J Psych. 1997; 171:364-8.

19. Gureje O. Psychiatry in Africa: the myths, the exotic, and the realities. Afr J Psychiatry (Johannesburg). 2007;10:11-4.

20. World Health Organization. International classification of diseases. 10th ed. Geneva: World Health Organization; 1992.

21. Fleiss JL. Statistical methods for rates and proportions. 2nd ed. New York: Wiley; 1981. p. 17.

22. Overall JE, Donald RG. The brief psychiatric rating scale. Psychol Rep. 1962;10:799-812.

23. Ladapo THO, Aina OF, Adebiyi OP, Olomu SO, Aina RB. Long stay patients in a psychiatric hospital in Lagos Nigeria. Afr J Psych. 2008;11:128-32.

24. Endicott J, Spitzer RL, Fleiss JL, Cohen J. The global assessment scale. A procedure for measuring overall severity of psychiatric disturbance. Arch Gen Psychiatry. 1976;33:766-71.

25. SPSS 16.0. SPSS Inc., 233 South wacker drive, 11th floor, Chicago. 2007.

26. Ascher-Svanum H, Novick D, Haro JM, Aguado J, Cui Z. Empirically driven definitions of "good', "'moderate', and "poor" levels of functioning in the treatment of schizophrenia. Qual Life Res. 2013;22:2085-94.

27. Johnson S, Sathyaseelan M, Charles H, Jeyaseelan V, Jacob KS. Insight, psychopathology, explanatory models and outcome of schizophrenia in India: a prospective 5-year cohort study. BMC Psychiatry. 2012;12:159.

28. Gelder M, Harrison P, Cowen P. Development of schizophrenia concepts and terminologies. In: Gelder M, Gath D, Mayou R, Cowen P, editors. Shorter Oxford text book of psychiatry. Oxford: Oxford University Press; 2008. p. 274-5.

29. Ukpong DI. Burden and psychological distress among Nigerian family caregivers of schizophrenic patients: the role of positive and negative symptoms. Turk J Psych. 2011;22:1-6.

30. Lasebikan VO. Validation of Yoruba version of family burden interview schedule (Y-FBIS) on caregivers of schizophrenia patients. ISRN Psychiatry. 2012;2012:165179.

31. Yusuf A, Nuhu F, Akinbiyi A. Caregiver burden among relatives of patients with schizophrenia in Katsina, Nigeria. South Afr J Psychiatry. 2009;15(2):43-7.

32. Kapungwe A, Cooper S, Mwanza J, Mwape L, Sikwese A, Kakuma R, Lund C, Flisher AJ. Mental illness-stigma and discrimination in Zambia. Afr J Psychiatry. 2010;13:192-203.

33. National Bureau of Statistics (NBS). Federal republic of Nigeria. Unemployment rate in Nigeria from section on employment and labour. Abuja: NBS; 1981. p. 17.

34. Crow TJ. Schizophrenia: diagnostic boundaries, epidemiology and brain changes. Clinician. 1993;11:2-17.

35. Wing JK. Mental illness. In: Steven A, Raftery J, editors. Epidemiology needs assessment. London: HMSO; 1994.

36. Hosseini SH, Karkhaneh YM. Quality of Life and GAF in schizophrenia correlation between quality of life and global functioning in schizophrenia. Iran J Psychiatry Behav Sci. 2011;5(2):120-5.

37. Crumlish N, Samalani P, Sefasi A, Kinsella A, O'Callaghan CE. Insight, psychopathology and global functioning in schizophrenia in urban Malawi. Br J Psychiatry. 2007;191:262-3.

38. Lasebikan VO, Ayinde OO. Effects of psychopathology, functioning and anti-psychotic medication adherence on caregivers' burden in schizophrenia. Indian J Psychol Med. 2013;35(2):135-40.

39. Caron J, Mercier C, Diaz P, Martin A. Socio-demographic and clinical predictors of quality of life in patients with schizophrenia or schizophrenia affective disorder. Psychiatry Res. 2005;137:203-13.

40. Lawal RA, Suleiman GT, Onyenze B. Risperidone in the treatment of schizophrenia. Nigerian Med Practitioner. 2003;44:11-8. 